\title{
ISOTROPIC IMMERSIONS AND VERONESE MANIFOLDS
}

\author{
BY \\ T. ITOH (1) AND K. OGIUE (2)
}

\begin{abstract}
An $n$-dimensional Veronese manifold is defined as a minimal immersion of an $n$-sphere of curvature $n / 2(n+1)$ into an $\{n(n+3) / 2-1\}$ dimensional unit sphere. The purpose of this paper is to give some characterizations of a Veronese manifold in terms of isotropic immersions.
\end{abstract}

1. Introduction. A Veronese surface may be characterized from a differential-geometric point of view as a minimal immersion of a 2-dimensional sphere of curvature $1 / 3$ into a 4-dimensional unit sphere. As a generalization of a Veronese surface, a Veronese manifold is defined as a minimal immersion of an $n$-dimensional sphere of curvature $n / 2(n+1)$ into an $\{n+n(n+1) / 2-1\}$ dimensional unit sphere.

The minimal immersions of a sphere into a sphere are completely determined by doCarmo and Wallach [2], among which a Veronese manifold can be considered as the simplest one.

On the other hand, O'Neill [5] defined a notion of isotropic immersions. The purpose of this paper is to provide some characterizations of a Veronese manifold in terms of isotropic immersions.

2. Preliminaries. A Riemannian manifold of constant curvature is called a space form. We denote by $M^{m}(c)$ an $m$-dimensional space form of constant curvature $c$.

Let $M$ be an $n$-dimensional Riemannian manifold isometrically immersed in $M^{n+p}(\widetilde{c})$. Then the second fundamental form $\sigma$ of the immersion is given by $\sigma(X, Y)=\widetilde{\nabla}_{X} Y-\nabla_{X} Y$ and it satisfies $\sigma(X, Y)=\sigma(Y, X)$.

We choose a local field of orthonormal frames $e_{1}, \cdots, e_{n}, e_{n+1}, \cdots$, $e_{n+p}$ in $M^{n+p}(\mathcal{C})$ in such a way that, restricted to $M, e_{1}, \cdots, e_{n}$ are tangent to $M$. With respect to the frame field of $M^{n+p}(\mathcal{c})$ chosen above, let $\omega^{1}, \cdots$, $\omega^{n}, \omega^{n+1}, \cdots, \omega^{n+p}$ be the field of dual frames. Then the structure

Received by the editors March 19, 1974.

AMS (MOS) subject classifications (1970). Primary 53C40.

(1) Partially supported by the Sakko-kai Foundation.

(2) Partially supported by the Matsunaga Science Foundation. 
equations of $M^{n+p}(\widetilde{c})$ are given by ( $\left.{ }^{3}\right)$

$$
\begin{gathered}
d \omega^{A}=-\sum \omega_{B}^{A} \wedge \omega^{B}, \quad \omega_{B}^{A}+\omega_{A}^{B}=0, \\
d \omega_{B}^{A}=-\sum \omega_{C}^{A} \wedge \omega_{B}^{C}+\tilde{c} \omega^{A} \wedge \omega^{B} .
\end{gathered}
$$

Restricting these forms to $M$, we obtain the structure equations of the immersion:

$$
\begin{gathered}
\omega^{\alpha}=0, \\
d \omega^{i}=-\sum \omega_{j}^{i} \wedge \omega^{j}, \quad \omega_{j}^{i}+\omega_{i}^{j}=0, \\
d \omega_{j}^{i}=-\sum \omega_{k}^{i} \wedge \omega_{j}^{k}+\Omega_{j}^{i}, \quad \Omega_{j}^{i}=\frac{1}{2} \sum R_{j k l}^{i} \omega^{k} \wedge \omega^{l} .
\end{gathered}
$$

From (2.1) and (2.3) it follows that $\sum \omega_{i}^{\alpha} \wedge \omega^{i}=0$. Therefore by Cartan's lemma, we may write

$$
\omega_{i}^{\alpha}=\sum h_{i j}^{\alpha} \omega^{j}, \quad h_{i j}^{\alpha}=h_{j i}^{\alpha} .
$$

The second fundamental form $\sigma$ and $h_{i j}^{\alpha}$ are related by $\sigma\left(e_{i}, e_{j}\right)=\sum h_{i j}^{\alpha} e_{\alpha}$ or $\sigma=$ $\sum h_{i j}^{\alpha} \omega^{i} \otimes \omega^{j} \otimes e_{\alpha}$. The equation of Gauss is given by

$$
R_{j k l}^{i}=\tilde{c}\left(\delta_{k}^{i} \delta_{j l}-\delta_{l}^{i} \delta_{j k}\right)+\sum\left(h_{i k}^{\alpha} h_{j l}^{\alpha}-h_{i l}^{\alpha} h_{j k}^{\alpha}\right) .
$$

Let $R_{i j}=\sum R_{i k j}^{k}$ and $\rho$ be the Ricci tensor and the scalar curvature of $M$, respectively. Then they satisfy

$$
\begin{gathered}
R_{i j}=(n-1) \tilde{c} \delta_{i j}+\sum\left(h_{k k}^{\alpha} h_{i j}^{\alpha}-h_{i k}^{\alpha} h_{k j}^{\alpha}\right), \\
\rho=n(n-1) \tilde{c}+\sum h_{i i}^{\alpha} h_{j j}^{\alpha}-\|\sigma\|^{2},
\end{gathered}
$$

where $\|\sigma\|$ denotes the length of the second fundamental form so that $\|\sigma\|^{2}=$ $\sum h_{i j}^{\alpha} h_{i j}^{\alpha}$. In particular, if the immersion is minimal, then they satisfy

$$
\begin{gathered}
R_{i j}=(n-1) \tilde{c} \delta_{i j}-\sum h_{i k}^{\alpha} h_{k j}^{\alpha}, \\
\rho=n(n-1) \tilde{c}-\|\sigma\|^{2} .
\end{gathered}
$$

Let $K_{N}=\sum\left(h_{i k}^{\alpha} h_{k j}^{\beta}-h_{i k}^{\beta} h_{k j}^{\alpha}\right)\left(h_{j l}^{\beta} h_{l i}^{\alpha}-h_{j l}^{\alpha} h_{l i}^{\beta}\right)$. Then $K_{N}$ is a nonnegative scalar on $M$ and is a geometric invariant.

If we define $h_{i j k}^{\alpha}$ by

(3) We use the following convention on the range of indices unless otherwise stated: $A, B, C, D=1, \cdots, n, n+1, \cdots, n+p ; i, j, k, l=1, \cdots, n ; \alpha, \beta=n+1, \cdots, n+p$, and we agree that repeated indices under a summation sign without indication are summed over the respective range. 


$$
\sum h_{i j k}^{\alpha} \omega^{k}=d h_{i j}^{\alpha}-\sum h_{k j}^{\alpha} \omega_{i}^{k}-\sum h_{i k}^{\alpha} \omega_{j}^{k}+\sum h_{i j}^{\beta} \omega_{\beta}^{\alpha},
$$

then from (2.2), (2.3) and (2.6) we have $h_{i j k}^{\alpha}=h_{i k j}^{\alpha}$. Let $\nabla^{\prime}$ be the covariant differentiation with respect to the connection in (tangent bundle) $\oplus$ (normal bundle). Then it follows that $\nabla^{\prime} \sigma=\Sigma h_{i j k}^{\alpha} \omega^{i} \otimes \omega^{j} \otimes \omega^{k} \otimes e_{\alpha}$.

Let $\mathfrak{G}=n^{-1} \Sigma \sigma\left(e_{i}, e_{i}\right)$ be the mean curvature vector and $H=\|\mathscr{G}\|$ be the mean curvature. Then it is known that the second fundamental form of the immersion satisfies a differential equation, that is, we have

LEMMA 2.1 [1]. If $M$ is an n-dimensional submanifold immersed in $M^{n+p}(\widetilde{c})$, then

$$
\begin{aligned}
1 / 2 \Delta\|\sigma\|^{2} & =\left\|\nabla^{\prime} \sigma\right\|^{2}+\sum h_{i j}^{\alpha} \Delta h_{i j}^{\alpha} \\
& =\left\|\nabla^{\prime} \sigma\right\|^{2}-K_{N}-\sum h_{i j}^{\alpha} h_{i j}^{\beta} h_{k l}^{\alpha} h_{k l}^{\beta}+n\left(\widetilde{c}+H^{2}\right)\|\sigma\|^{2}-n^{2} \widetilde{c} H^{2}+n H \Delta H,
\end{aligned}
$$

where $\Delta$ denotes the Laplacian.

3. Isotropic submanifolds. For a unit vector $X, \sigma(X, X)$ is called the normal curvature vector in the direction of $X$. An isometric immersion is said to be isotropic if every normal curvature vector has the same length at each point, that is, the length of the normal curvature vector depends only on the point. In particular, if the length of the normal curvature vector is equal to $\lambda$ (a function on the submanifold), then the immersion is said to be $\lambda$-isotropic. The following two lemmas are due to B. O'Neill [5].

LEMmA 3.1. An isometric immersion is isotropic if and only if $\langle\sigma(X, X)$, $\sigma(X, Y)\rangle=0$ for all orthogonal vectors $X$ and $Y$.

LEMma 3.2. A $\lambda$-isotropic immersion satisfies

(i) $\langle\sigma(X, X), \sigma(Y, Y)\rangle+2\|\sigma(X, Y)\|^{2}=\lambda^{2}$,

(ii) $\langle\sigma(X, X), \sigma(U, V)\rangle+2\langle\sigma(X, U), \sigma(X, V)\rangle=0$,

(iii) $\langle\sigma(X, Y), \sigma(U, V)\rangle+\langle\sigma(X, U), \sigma(Y, V)\rangle+\langle\sigma(X, V), \sigma(Y, U)\rangle=0$, for orthonormal vectors $X, Y, U, V$.

4. Isotropic minimal submanifolds. In this section we consider isotropic minimal submanifolds immersed in a space form and give a characterization of a Veronese manifold.

If $M$ is an $n$-dimensional $\lambda$-isotropic minimal submanifold, then it follows easily from Lemma 3.2 (i), (ii) that

$$
\sum h_{i k}^{\alpha} h_{k j}^{\alpha}=1 / 2(n+2) \lambda^{2} \delta_{i j} .
$$

This, combined with $(2.8)_{m}$, implies the following: 
Proposition 4.1. Let $M$ be an n-dimensional $\lambda$-isotropic minimal submanifold immersed in $M^{n+p}(\widetilde{c})$. If $n \geqslant 3$, then $M$ is Einstein

$$
\left(R_{i j}=\left\{(n-1) \tilde{c}-1 / 2(n+2) \lambda^{2}\right\} \delta_{i j}\right)
$$

so that $\lambda$ is constant.

We shall prove several lemmas.

LEMMA 4.1. If $M$ is an n-dimensional isotropic minimal submanifold immersed in $M^{n+p}(\widetilde{c})$, then

$$
K_{N}=\frac{2}{n+2}\|\sigma\|^{4}+\sum h_{i j}^{\alpha} h_{i j}^{\beta} h_{k l}^{\alpha} h_{k l}^{\beta} .
$$

Proof. From (4.1) we have

$$
\|\sigma\|^{2}=\sum h_{i j}^{\alpha} h_{i j}^{\alpha}=1 / 2 n(n+2) \lambda^{2} .
$$

The definition of $K_{N}$, together with (4.1) and (4.2), yields

$$
K_{N}=2 n^{-1}\|\sigma\|^{4}-2 \sum h_{i k}^{\alpha} h_{k j}^{\beta} h_{j i}^{\alpha} h_{l i}^{\beta} \text {. }
$$

On the other hand, Lemma 3.1 and Lemma 3.2 imply

$$
\begin{gathered}
\sum_{\alpha} h_{i i}^{\alpha} h_{i i}^{\alpha}=\lambda^{2}, \quad \sum_{\alpha} h_{i i}^{\alpha} h_{i j}^{\alpha}=0, \quad \sum_{\alpha} h_{i i}^{\alpha} h_{j l}^{\alpha}+2 \sum_{\alpha} h_{i j}^{\alpha} h_{i l}^{\alpha}=0, \\
\sum_{\alpha} h_{i i}^{\alpha} h_{j j}^{\alpha}+2 \sum_{\alpha} h_{i j}^{\alpha} h_{i j}^{\alpha}=\lambda^{2}, \\
\sum_{\alpha} h_{i j}^{\alpha} h_{k l}^{\alpha}+\sum_{\alpha} h_{i k}^{\alpha} h_{j l}^{\alpha}+\sum_{\alpha} h_{i l}^{\alpha} h_{j k}^{\alpha}=0,
\end{gathered}
$$

where $i, j, k, l$ are distinct. Therefore we can see that

$$
\begin{aligned}
\sum h_{i k}^{\alpha} h_{k j}^{\beta} h_{j l}^{\alpha} h_{l i}^{\beta}= & -\frac{1}{2} \sum_{i, j, k, l \neq}\left(\sum_{\alpha, \beta} h_{i j}^{\alpha} h_{i j}^{\beta} h_{k l}^{\alpha} h_{k l}^{\beta}\right) \\
& -\frac{3}{2} \sum_{i, j, l \neq}\left(\sum_{\alpha, \beta} h_{i i}^{\alpha} h_{i i}^{\beta} h_{j l}^{\alpha} h_{j l}^{\beta}\right)+\sum_{i \neq j}\left(\sum_{\alpha, \beta} h_{i j}^{\alpha} h_{i j}^{\beta} h_{i j}^{\alpha} h_{i j}^{\beta}\right) \\
& +2 \sum_{i \neq j}\left(\sum_{\alpha, \beta} h_{i j}^{\alpha} h_{i i}^{\beta} h_{i j}^{\alpha} h_{j i}^{\beta}\right)+n \lambda^{4} ; \\
\sum h_{i j}^{\alpha} h_{i j}^{\beta} h_{k l}^{\alpha} h_{k l}^{\beta}= & \sum_{i, j, k, l \neq}\left(\sum_{\alpha, \beta} h_{i j}^{\alpha} h_{i j}^{\beta} h_{k l}^{\alpha} h_{k l}^{\beta}\right)+3 \sum_{i, k, l \neq}\left(\sum_{\alpha, \beta} h_{i i}^{\alpha} h_{i i}^{\beta} h_{k l}^{\alpha} h_{k l}^{\beta}\right) \\
& +2 \sum_{i \neq j}\left(\sum_{a, \beta} h_{i j}^{\alpha} h_{i j}^{\beta} h_{i j}^{\alpha} h_{i j}^{\beta}\right)+\sum_{i \neq j}\left(\sum_{\alpha, \beta} h_{i i}^{\alpha} h_{i i}^{\beta} h_{i j}^{\alpha} h_{j j}^{\beta}\right)+n \lambda^{4},
\end{aligned}
$$

where $\sum_{i, j, k, l \neq}$ denotes the summation over distinct $i, j, k, l$. It follows from (4.2) and (4.6) that 


$$
\begin{aligned}
K_{N} & =\frac{2}{n}\|\sigma\|^{4}+\sum h_{i j}^{\alpha} h_{k l}^{\alpha} h_{i j}^{\beta} h_{k i}^{\beta}-\frac{4\|\sigma\|^{4}}{n(n+2)} \\
& =\frac{2\|\sigma\|^{4}}{n+2}+\sum h_{i j}^{\alpha} h_{k l}^{\alpha} h_{i j}^{\beta} h_{k l}^{\beta} . \quad \text { Q.E.D. }
\end{aligned}
$$

LEMMA 4.2. Let $M$ be an n-dimensional isotropic minimal submanifold immersed in $M^{n+p}(\widetilde{c})$. Then

$$
K_{N} \geqslant \frac{2 n}{(n+2)(n-1)}\|\sigma\|^{4} .
$$

The equality holds if and only if $M$ is a space form.

Proof. From (4.1), $\cdots,(4.5)$, we have

$$
\begin{aligned}
K_{N}= & \frac{2}{n}\|\sigma\|^{4}-\frac{8\|\sigma\|^{4}}{n(n+2)^{2}}+\sum_{i, j, k, l \neq}\left(\sum_{\alpha, \beta} h_{i j}^{\alpha} h_{k l}^{\alpha} h_{i j}^{\beta} h_{k l}^{\beta}\right) \\
& +3 \sum_{i, j, k \neq}\left(\sum_{\alpha, \beta} h_{i i}^{\alpha} h_{j k}^{\alpha} h_{i i}^{\beta} h_{j k}^{\beta}\right)-2 \sum_{i \neq j}\left(\sum_{\alpha, \beta} h_{i j}^{\alpha} h_{i j}^{\alpha} h_{i j}^{\beta} h_{i j}^{\beta}\right) \\
& -4 \lambda^{2} \sum_{i \neq j}\left(\sum_{\alpha} h_{i j}^{\alpha} h_{i j}^{\alpha}\right)+8 \sum_{i \neq j}\left(\sum_{\alpha, \beta} h_{i j}^{\alpha} h_{i j}^{\alpha} h_{i j}^{\beta} h_{i j}^{\beta}\right) \\
\geqslant & \frac{2(n+4)}{(n+2)^{2}}\|\sigma\|^{4}-\frac{8\|\sigma\|^{4}}{(n+2)^{2}}+6 \sum_{i \neq j}\left(\sum_{\alpha, \beta} h_{i j}^{\alpha} h_{i j}^{\alpha} h_{i j}^{\beta} h_{i j}^{\beta}\right) \\
= & \frac{2 n\|\sigma\|^{4}}{(n+2)^{2}}+6 \sum_{i \neq j}\left\{\sum_{\alpha} h_{i j}^{\alpha} h_{i j}^{\alpha}-\frac{n \lambda^{2}}{2(n-1)}\right\}^{2}+\frac{3 n^{3}}{2(n-1)} \lambda^{4} \\
\geqslant & \frac{2 n\|\sigma\|^{4}}{(n+2)(n-1)} .
\end{aligned}
$$

The equality holds if and only if

$$
\begin{gathered}
\sum_{\alpha, \beta} h_{i j}^{\alpha} h_{k l}^{\alpha} h_{i j}^{\beta} h_{k l}^{\beta}=0, \quad \sum_{\alpha} h_{i i}^{\alpha} h_{j k}^{\alpha}=0, \\
\sum_{\alpha} h_{i j}^{\alpha} h_{i j}^{\alpha}=\frac{n \lambda^{2}}{2(n-1)}
\end{gathered}
$$

holds for distinct $i, j, k, l$.

If (4.7) holds, then from (2.7), (4.4) and (4.7) it follows that

$$
R_{j k l}^{i}=\left(\tilde{c}-\frac{(n+2) \lambda^{2}}{2(n-1)}\right)\left(\delta_{k}^{i} \delta_{j l}-\delta_{l}^{i} \delta_{j k}\right)
$$

so that $M$ is a space form. Q.E.D.

We are now in a position to prove the following: 
THEOREM 4.1. Let $M$ be an n-dimensional $\lambda$-isotropic minimal submanifold immersed in $M^{n+p}(\widetilde{c}), \tilde{c}>0$. If

(i) $n \geqslant 3$,

(ii) $\lambda>0$,

(iii) $K_{N} \leqslant(n \tilde{c} /(n+1))\|\sigma\|^{2}+(2 /(n+2))\|\sigma\|^{4}$,

(iv) the immersion is full,

then $M$ is a space form of constant curvature $n \widetilde{c} / 2(n+1)$ and $p=n(n+1) / 2-1$ so that $M$ is immersed as a Veronese manifold.

Proof. It follows from Lemma 2.1, Proposition 4.1 and Lemma 4.1 that

$$
\begin{aligned}
\left\|\nabla^{\prime} \sigma\right\|^{2} & =2 K_{N}-\frac{2}{n+2}\|\sigma\|^{4}-n \widetilde{c}\|\sigma\|^{2} \\
& =K_{N}-\frac{n^{2} \widetilde{c}}{n+1}\|\sigma\|^{2}+K_{N}-\frac{n \widetilde{c}}{n+1}\|\sigma\|^{2}-\frac{2}{n+2}\|\sigma\|^{4} .
\end{aligned}
$$

On the other hand, assumption (iii) and Lemma 4.2 imply that $K_{N} \leqslant$ $\left(n^{2} \widetilde{c} /(n+1)\right)\|\sigma\|^{2}$. This, together with (4.8) and assumption (iii), yields $\nabla^{\prime} \sigma=0$ and

$$
K_{N}=\frac{n^{2} \widetilde{c}}{n+1}\|\sigma\|^{2}=\frac{n \tilde{c}}{n+1}\|\sigma\|^{2}+\frac{2}{n+2}\|\sigma\|^{4}
$$

Hence

$$
\|\sigma\|^{2}=\frac{n(n-1)(n+2)}{2(n+1)} \widetilde{c} \text { and } K_{N}=\frac{n^{3}(n-1)(n+2)}{2(n+1)^{2}} \widetilde{c}^{2}
$$

so that

$$
K_{N}=\frac{2 n}{(n+2)(n-1)}\|\sigma\|^{4} .
$$

Therefore it follows from Lemma 4.2 that $M$ is a space form. This, combined with assumption (iv), Theorem in [3] and Theorem 1 in [4], completes the proof. Q.E.D.

5. Isotropic immersion of a space form into a space form. In this section we consider an $n$-dimensional space form $M^{n}(c)$ immersed in $M^{n+p}(\widetilde{c})$. The following lemma is due to O'Neill [5].

LEMMA 5.1. If $M^{n}(c)$ is a $\lambda$-isotropic submanifold immersed in $M^{n+p}(\widetilde{c})$, then

(1) $3\|\sigma(X, Y)\|^{2}+c-\tilde{c}=\lambda^{2}$,

(2) $2(c-\widetilde{c})+\lambda^{2}=3\langle\sigma(X, X), \sigma(Y, Y)\rangle$,

(3) $\langle\sigma(X, Y), \sigma(Z, U)\rangle_{.}=\langle\sigma(X, X), \sigma(Y, Z)\rangle=\langle\sigma(X, Y), \sigma(X, Z)\rangle=0$, for orthonormal $X, Y, Z, U$. Moreover if $-((n+2) / 2(n-1)) \lambda^{2}<c-\widetilde{c}<\lambda^{2}$, then $p \geqslant n(n+1) / 2$. 
From Lemma 5.1 we have

$$
\sum_{\alpha} h_{i i}^{\alpha} h_{i i}^{\alpha}=\lambda^{2}, \quad 3 \sum_{\alpha} h_{i j}^{\alpha} h_{i j}^{\alpha}+c-\tilde{c}=\lambda^{2}
$$

$$
\begin{gathered}
2(c-\tilde{c})+\lambda^{2}=3 \sum_{\alpha} h_{i i}^{\alpha} h_{j j}^{\alpha}, \\
\sum_{\alpha} h_{i j}^{\alpha} h_{k l}^{\alpha}=\sum_{\alpha} h_{i i}^{\alpha} h_{j l}^{\alpha}=\sum_{\alpha} h_{i j}^{\alpha} h_{i l}^{\alpha}=0,
\end{gathered}
$$

where all indices are distinct. It follows from (4.5), (4.6) and (5.1) that

$$
\begin{gathered}
K_{N}=(2 n / 9)\left\{(n+2) \lambda^{2}-(n-1)(c-\widetilde{c})\right\}^{2}-(2 n / 9)(n-1)\left(\lambda^{2}-c+\widetilde{c}\right)^{2} \\
-(4 n / 9)(n-1)\left(\lambda^{2}-c+\widetilde{c}\right)\left(\lambda^{2}+2 c-2 \widetilde{c}\right)-2 n \lambda^{4} \\
\sum h_{i j}^{\alpha} h_{k l}^{\alpha} h_{i j}^{\beta} h_{k l}^{\beta}=(2 n / 9)(n-1)\left(\lambda^{2}-c+\widetilde{c}\right)^{2} \\
+(n / 9)(n-1)\left(\lambda^{2}+2 c-2 \widetilde{c}\right)^{2}+n \lambda^{4}
\end{gathered}
$$

The following is easily seen.

PROPOSITION 5.1. If $M^{n}(c)$ is an isotropic submanifold immersed in $M^{n+p}(\widetilde{c})$, then $M^{n}(c)$ is a pseudo-umbilical submanifold in $M^{n+p}(\widetilde{c})$.

Finally we prove the following.

THEOREM 5.1. Let $M^{n}(c)$ be an n-dimensional compact oriented space form, which is isotropically immersed in $M^{n+p}(\widetilde{c})$. If

(i) $0<H^{2} \leqslant(2(n+1) / n) c-\tilde{c}$,

(ii) the immersion is full and $p>1$, then $H^{2}=(2(n+1) / n) c-\widetilde{c}$ and $p=n(n+1) / 2$. Moreover $M^{n}(c)$ is immersed as a Veronese manifold in some totally umbilical hypersurface of $M^{n+p}(\widetilde{c})$.

Proof. Since $H>0$ on $M^{n}(c)$, in view of Proposition 5.1, we can choose $e_{n+1}=H^{-1}$ g so that

$$
h_{i j}^{n+1}=H \delta_{i j} \text {. }
$$

Let $s=\Sigma_{\beta>n+1} h_{i j}^{\beta} h_{i j}^{\beta}=\|\sigma\|^{2}-n H^{2}=n(n-1)\left(H^{2}-c+\widetilde{c}\right)$. Then, using (5.6), we get 


$$
\begin{aligned}
1 / 2 \Delta s & =1 / 2 \Delta\|\sigma\|^{2}-n\|d H\|^{2}-n H \Delta H \\
& =\left\|\nabla^{\prime} \sigma\right\|^{2}+\sum h_{i j}^{\alpha} \Delta h_{i j}^{\alpha}-n\|d H\|^{2}-n H \Delta H \\
& =\sum h_{i j}^{\alpha} \Delta h_{i j}^{\alpha}+\sum_{\beta>n+1}\left(h_{i j k}^{\beta}\right)^{2}-n H \Delta H+\sum_{i \neq j}\left(h_{i j k}^{n+1}\right)^{2} .
\end{aligned}
$$

On the other hand, using Lemma $2.1,(5.4)$ and (5.5), we have

$$
\begin{aligned}
\sum h_{i j}^{\alpha} \Delta h_{i j}^{\alpha}= & n H \Delta H+n\left(\widetilde{c}+H^{2}\right)\|\sigma\|-n^{2} \widetilde{c} H^{2}-n^{2} H^{4} \\
& -(2 / 9)(n-1)(n+1)(n+2)\left(\lambda^{2}-c+\widetilde{c}\right)^{2} .
\end{aligned}
$$

Since

$$
\lambda^{2}-(c-\widetilde{c})=\frac{3 n}{n+2}\left(H^{2}-c+\widetilde{c}\right)=\frac{3}{(n-1)(n+2)} s,
$$

it follows from (5.7) and (5.8) that

$$
\begin{aligned}
1 / 2 \Delta s & =\sum_{\beta>n+1}\left(h_{i j k}^{\beta}\right)^{2}+n\left(\widetilde{c}+H^{2}\right) s-\frac{2(n+1)}{(n+2)(n-1)} s^{2}+\sum_{i \neq j}\left(h_{i j k}^{n+1}\right)^{2} \\
& =\sum_{\beta>n+1}\left(h_{i j k}^{\beta}\right)^{2}+\frac{n^{2} s}{n+2}\left\{\frac{2(n+1)}{n} c-\tilde{c}-H^{2}\right\}+\sum_{i \neq j}\left(h_{i j k}^{n+1}\right)^{2}
\end{aligned}
$$

Since $M^{n}(c)$ is compact, oriented and $(2(n+1) / n) c-\widetilde{c}-H^{2} \geqslant 0$, we have

$$
h_{i j k}^{\beta}=0 \text { for } \beta>n+1 \text { and } h_{i j k}^{n+1}=0 \text { for } i \neq j
$$

and

$$
s=0 \text { or } H^{2}=(2(n+1) / n) c-\widetilde{c} .
$$

If $s=0$, then $M^{n}(c)$ is totally umbilical, so that $p=1$, which contradicts the assumption. Hence $H^{2}=(2(n+1) / n) c-\widetilde{c}$, which implies that $\|\sigma\|^{2}$ and $s$ are constant. Since $H>0$ and $s>0,(5.2)$ and (5.9) imply $-((n+2) / 2(n-1)) \lambda^{2}<$ $c-\widetilde{c}<\lambda^{2}$ so that $p \geqslant n(n+1) / 2$. Moreover it follows from (5.7) and (5.10) that $\Sigma h_{i j}^{\alpha} \Delta h_{i j}^{\alpha}=0$ which, together with Lemma 2.1 , implies that $\nabla^{\prime} \sigma=0$. Therefore, by Theorem in [3] , $p=n(n+1) / 2$.

Furthermore it follows easily from $\nabla^{\prime} \sigma=0$ that the mean curvature vector 5 is parallel. This combined with Proposition 5.1, implies that $M^{n}(c)$ is immersed in some totally umbilical hypersurface of $M^{n+p}(\widetilde{c})$ as a minimal submanifold and hence must be a Veronese manifold. Q.E.D.

\section{BIBLIOGRAPHY}

1. S. S. Chern, M. do Carmo and S. Kobayashi, Minimal submanifolds of a sphere with second fundamental form of constant length, Functional Analysis and Related Fields (Proc. Conf. for M. Stone, Univ. Chicago, Chicago, Ill., 1968), Springer, New York, 1970, pp. 59-75. MR 42 \#8424.

2. M.P. do Carmo and N. R. Wallach, Minimal immersions of spheres into spheres, Ann. of Math. (2) 93 (1971), 43-62. MR 43 = 4048 . 
3. J. A. Erbacher, Reduction of the codimension of an isometric immersion, J. Differential Geometry 5 (1971), 333-340. MR 44 \#5897.

4. T. Itoh and K. Ogiue, Isotropic immersions, J. Differential Geometry 8 (1973), $305-316$.

5. B. O'Neill, Isotropic and Kähler immersions, Canad. J. Math. 17 (1965), 907915. MR $32 \# 1654$.

DEPARTMENT OF MATHEMATICS, TOKYO UNIVERSITY OF EDUCATION, 3-29-1 OTSUKA, BUNKYO-KU, TOKYO, JAPAN

DEPARTMENT OF MATHEMATICS, MICHIGAN STATE UNIVERSITY, EAST LANSING, MICHIGAN 48823

Current address (T. Itoh): Department of Mathematics, Tsukuba University, Ibaragi, 300-31, Japan

Current address (K. Ogiue): Department of Mathematics, Tokyo Metropolitan University, Setagaya, Tokyo, 158, Japan 